\title{
Polyethylene Glycol Backfilling Mitigates the Negative Impact of the Protein Corona on Nanoparticle Cell Targeting
}

\author{
Qin Dai, Carl Walkey, and Warren C. W. Chan
}

Version Post-Print/Accepted Manuscript

Citation Q. Dai, C. D. Walkey, W. C. W. Chan, "Polyethylene Glycol Backfilling (published version) Mitigates the Negative Impact of the Protein Corona on Nanoparticle Cell Targeting," Angewandte Chemie International Edition, 2014 (Apr), 53, 5093-5096. JIF $=13.7$.

Publisher's Statement This is the peer reviewed version of the following article: Q. Dai, C. D. Walkey, W. C. W. Chan, "Polyethylene Glycol Backfilling Mitigates the Negative Impact of the Protein Corona on Nanoparticle Cell Targeting," Angewandte Chemie International Edition, 2014 (Apr), 53, 5093-5096. JIF = 13.7., which has been published in final form at http://dx.doi.org/10.1002/anie.201309464. This article may be used for non-commercial purposes in accordance with Wiley Terms and Conditions for Self-Archiving.

\section{How to cite TSpace items}

Always cite the published version, so the author(s) will receive recognition through services that track citation counts, e.g. Scopus. If you need to cite the page number of the TSpace version (original manuscript or accepted manuscript) because you cannot access the published version, then cite the TSpace version in addition to the published version using the permanent URI (handle) found on the record page. 
DOI : $10.1002 /$ anie.201309464

Communication

\section{Polyethyleneglycol Backfilling Mitigates the Impact of the Protein Corona on Targeting**}

Qin Dai, [a] Carl Walkey, [a] Prof. Warren^^${ }^{\wedge} C .{ }^{\wedge} W$. Chan*[a]

[a] Institute of Biomaterials and Biomedical Engineering, University of Toronto

Toronto, M5S 3 G9 (Canada)

E-mail: warren.chaneutoronto.ca

${ }^{[* *]}$ We would like to thank Conrad Chan and Dr. Raymond Reilly for generously providing us with the SKBR3 cells. We would also like to thank Dr. Christopher Yip for the use of the confocal microscope. Lastly, we would like to thank Edward^^A. Sykes for providing feedback and ideas.

<pict> Supporting information for this article is available on the WWW under <url>http://dx.doi.org/10.1002/anie.201309464</url>.

Maß halten: Die Adsorption von Serumproteinen (farbige Knäuel) an zellspezifische Nanopartikeln (orangefarbene Kreise) kann eine unspezifische Zellbindung nach sich ziehen (rote Pfeile). Polyethylenglycol (PEG; geschwungene graue Linien) kann dies verhindern; lange PEG-Ketten stören aber auch die spezifische Bindung am gewünschten Angriffspunkt (grüne Pfeile), und nur kürzere PEG-Ketten unterbinden gezielt die unspezifische Bindung . $<$ ? $><$ ? $>$ ok? $<$ ? $><$ ? $>$

Spezifische Nanopartikel

Nanopartikel

Oberflächenchemie

PEGylierung

Polymere

Zellspezifität 
The long and short of it: The adsorption of serum proteins (colored knots) onto targeted nanoparticles (orange circles) can lead to nonspecific cell binding (solid red arrows) . Polyethylene glycol (PEG; grey curves) can be used to prevent this; however, long PEG chains can disrupt specific target binding. Shorter PEG chains prevent nonspecific binding without interfering with target recognition (solid green arrow). $<$ ? $><$ ? > ok? $<$ ? $><$ ? $>$

Nanoparticle Targeting

cell targeting

nanoparticles

PEGylation

polymers

surface chemistry

$<$ ? $><$ ? $>$ Title ok? $<$ ? $><$ ? $>$ In protein-rich environments such as the blood, the formation of a protein corona on receptor-targeting nanoparticles prevents target recognition. As a result, the ability of targeted nanoparticles to selectively bind to diseased cells is drastically inhibited. Here, we demonstrate that backfilling the surface of a targeted nanoparticle with polyethylene glycol (PEG) molecules reduces the formation of the protein corona and re-establishes specific binding. The length of the backfilled PEG molecules must be less than the length of the ligand linker; otherwise, PEG interferes with the binding of the targeting ligand to its corresponding cellular receptor.

$<?><?>0 \mathrm{k} ?<?><?>$

Nanoparticles are being developed for the diagnosis and treatment of a variety of diseases.[1] The surfaces of these nanoparticles are typically functionalized with antibodies, [2] peptides, [3] or aptamers[4] that enable them to recognize specific receptors on cell surfaces. This allows the nanoparticles to selectively accumulate in diseased tissues, which could enhance the diagnostic sensitivity or therapeutic index while preventing accumulation in off-target tissues. $<$ ? $><$ ? $>$ Sentence ok? $<$ ? $><$ ? $>$ In order to improve the efficiency of nanoparticle delivery into diseased tissues, researchers have focused on understanding how targeted nanoparticles interact with biological components such as blood, [5] tissues, [6] and cells. [7] An emerging body of literature demonstrates that when nanoparticles are introduced into the blood, serum proteins interact with the nanoparticle surface to form a protein corona. [8] These adsorbed proteins 
affect nanoparticle pathophysiology[9] and mask the ability of targeted nanoparticles to interact with cell receptors, thereby leading to a loss of targeting specificity. [10] To suppress protein adsorption, researchers often functionalize the nanoparticle surface with the antifouling polymer polyethylene glycol (PEG).[11] In order to properly design target-specific nanoparticles, a detailed understanding of the amount of PEG to apply and the type of PEG to use is essential to effectively reduce serum protein-nanoparticle interactions. $<$ ? $><$ ? $>$ Sentence ok? $<$ ? $><$ ? $>$ To date, it is unclear whether PEG backfilling can improve the specific binding of nanoparticles to a targeted cell population by overcoming the inhibitory effect of the protein corona. In this study, we investigated whether backfilling receptor-targeting nanoparticles with PEG could suppress serum protein adsorption and re-establish binding specificity.

$<$ ? $><$ ? $>$ Sentence ok? $<$ ? $><$ ? $>$

50^^nm gold nanoparticles functionalized with fluorescent Herceptin were synthesized for the purpose of this study (Figure^^1^A<figrl>, Table^^S1 in the Supporting Information). We used gold nanoparticles as a model system because of their ease of synthesis and[12] characterization, [13] and their relevance in targeted delivery. [14] Herceptin was chosen as the biorecognition molecule because of its prevalent use for the therapeutic and diagnostic targeting of the ErbB2<?><?>ErbB2? Change throughout? Yes, please. It has been changed<?><?> receptors in breast cancer. [15] Amines on the Herceptin molecule were labeled with the near-infrared-emitting dye Alexa Fluor 647 using amine-reactive succinimidyl ester chemistry. The high quantum yield and near-infrared emission of the Alexa Fluor dye minimizes gold surface quenching[16], making this fluorophore suitable for use as a fluorescent reporter for the nanoparticle. $<$ ? $><$ ? $>$ Sentence ok? $<$ ? $><$ ? $>$ UV/Vis spectrophotometric measurements confirmed that an average of 1.8 dye molecules were conjugated per Herceptin molecule. The low dye-to-protein ratio allows other primary amine groups on Herceptin to be further chemically modified with a $5^{\wedge \wedge} \mathrm{kDa}$ orthopyridyldisulfide polyethyleneglycol $N$-hydroxysuccinimide ester (OPSS-PEG-NHS ester, Figure^^1^A<xfigr1>). Previously, we found that this extra step in the nanoparticle-coating 
process increased the strength of ligand binding to the surface of the gold nanoparticle (unpublished data). A similar strategy was used by Chattopadhyay et^^al. to successfully conjugate Herceptin to gold nanoparticles. [17] To gauge the range in which the conjugated Herceptin retains binding specificity for the ErbB2 receptors, we performed cell-binding experiments with serially diluted OPSS-PEG-NHS ester conjugated to Herceptin. Upon treatment of ErbB2<m+> SKBR3 cells with these Herceptin conjugates, we found that the specific binding of Herceptin is preserved when there are fewer than 125 OPSS-PEG-NHS ester molecules added per Herceptin molecule (Figures^^s1, s2 in the Supporting Information). $<$ ? $><$ ? $>$ Sentence ok? $<$ ? $><$ ? $>$ Finally, the thiolated and fluorescently labeled Herceptin was incubated with gold nanoparticles at a molar ratio of $21: 1$, and approximately 16.5 Herceptin molecules were conjugated per nanoparticle (Table^^S1). To stabilize the nanoparticles and prevent protein adsorption, additional methoxy-terminated polythethyleneglycol (mPEG) molecules were added at $6 \mathrm{PEG} / \mathrm{nm}^{2}$ to fully saturate or "backfill" the remaining gold nanoparticle surface (Figure^^1^A<xfigr $1>$ ). The hydrodynamic size and surface charge of all of the nanoparticle designs was characterized (Table^^s1) .

In the first set of experiments, we characterized the ErbB2 receptor expression on ErbB2<m+> SKBR3 and ErbB2<m-> MCF7 cells by introducing an excess of unlabeled Herceptin to compete with labeled Herceptin for the ErbB2 receptors. We found that a 50-fold excess of unlabeled Herceptin was sufficient to effectively block the binding of the conjugated Herceptin (Figure^^s3). Next, we tested the binding specificity of the targeted nanoparticles to the SKBR3 cell line. The confocal image showed specific nanoparticle interaction with the membrane of the SKBR3 cells in the absence of competing ligands (Figure^^1^B<xfigr $1>$ ). The nanoparticles did not interact with the SKBR3 or MCF7 cells 
when the ErbB2 receptors were competitively bound by unlabeled Herceptin molecules (Figure^^ $1^{\wedge} B<x f i g r 1>$ ) . $<$ ? $><$ ? $>$ Sentence ok? $<$ ? $><$ ? $>$ Flow cytometry was also used to quantitatively determine the binding specificity of the nanoparticles. As a normalization procedure, the specificity index for nanoparticle--cell interaction was calculated as follows $[\mathrm{Eq} \cdot \wedge \wedge(1)<\mathrm{fffr} 1>]$ :

<fffl>Specificity index $=<$ brm $>$ Cell Binding $(<\mathrm{M}-$ $>$ competition) $<$ brtr $>$ Cell Binding (+competition) $</$ brm $><$ ZS $>$ (1)

This index acts as a numerical determinant of the ability of a nanoparticle to specifically interact with the ErbB2 receptor. Higher values indicate higher binding specificity. The Herceptin-conjugated nanoparticles have a specificity index of 4.67 and 1.02 for SKBR3 and MCF7 cells (Figure^^1^C<xfigrl>), respectively, showing that the nanoparticles specifically bind the ErbB2 receptor-expressing SkBR3 cells.<?><?>Sentence ok? Meaning was unclear.<?><?> Next, we determined whether serum-protein adsorptionreduces the specificity of Herceptin-conjugated nanoparticles for the ErbB2 receptor. Herceptin-conjugated nanoparticles were treated with $100^{\wedge} \circ$ human serum at $10^{\wedge} \wedge \mu \mathrm{L}^{\wedge} \mathrm{Cm}^{<\mathrm{M}->2}$ at $37^{\wedge}{ }^{\circ} \mathrm{C}$ (Figure^^1<xfigr1>, Figure^^S4) and were subsequently incubated with cells. These nanoparticles displayed visibly higher protein adsorption compared to nanoparticles not treated with human serum (Figure^^S5A). The nanoparticles with the adsorbed serum protein corona were calculated to have a specificity index of 1.50 and 1.00 for SKBR3 and MCF7 cells (Figure^^1^D<xfigr $1>$ ), respectively, indicating a drastic reduction in binding specificity compared to the nanoparticles that were not exposed to serum. This result shows that the adsorbed serum protein corona reduces the interaction of the Herceptin-conjugated nanoparticles with the ErbB2 receptor.<?><?>Sentence ok?<?><?> A similar trend was observed when the nanoparticles were exposed to 10^\% human serum 
(Figure^^S6). Furthermore, the protein-adsorbed nanoparticles did not show completely abolished total cell binding (Figure^^1^D<xfigr $1>$ ), a result that suggests that nanoparticle--cell interactions may occur through alternative pathways that are mediated by the adsorbed serum protein. Similar results were obtained by Salvati et^^al., where transferrin-targeted silica nanoparticles also experienced a reduction in binding specificity after exposure to serum.[1]

Once we had confirmed that serum protein effectively reduced the targeting specificity of Herceptin-conjugated nanoparticles towards ErbB $2<\mathrm{m}+>$ cells, we wanted to determine whether backfilling the nanoparticles with thiolated PEG rescues the targeting specificity of the nanoparticles after exposure to serum protein. PEG groups are known to reduce nonspecific protein adsorption. [5] We hypothesize that nucleation of the protein corona occurs when there are available sites on the nanoparticle for the binding of serum proteins, which can catalyze the growth of the corona. To address this problem, we saturated the remaining bare surface of the nanoparticles with thiol-terminated PEG groups, followed by incubation with human serum. The thiolated PEG is datively bound to the nanoparticle surface because of the strong binding strength of sulfur to gold atoms. For nanoparticles that do not permit thiol--metal interactions, other linking chemistries such as click or carbodiimide chemistry may be used for backfilling the nanoparticle surface with PEG.[18] PEG backfilling effectively suppressed the adsorption of serum proteins to the surface of the nanoparticles (Figures^^S5B,^S7). In the first set of experiments, we used a $2^{\wedge} \wedge \mathrm{kDa}$ thiol-terminated mPEG for backfilling the Herceptin-conjugated gold nanoparticles. When introduced to cells, these backfilled nanoparticles were shown to be 2.5-fold more responsive to SKBR3 cells than MCF7 cells, with specificity indices of 3.07 and 1.24

(Figure^^1^E<xfigrl>), respectively. This result suggests that mPEG backfilling can partially rescue the specificity of the nanoparticles for ErbB2 receptors after exposure to serum.

Next, we wanted to evaluate whether the chain length, which is a function of the molecular weight, $<$ ? $><$ ? > Ok? kDa can't be used as units of length. Chain length is a function of $M W<?><$ ? $>$ of the backfilled $\mathrm{PEG}$ is important for determining the specificity of the Herceptin-conjugated nanoparticles for ErbB $2<\mathrm{m}+>$ cells. We produced particles backfilled with PEG of molecular weight 1,5 , and $10^{\wedge} \wedge \mathrm{kDa}$. We first assessed the binding specificity of these nanoparticles prior to serum 


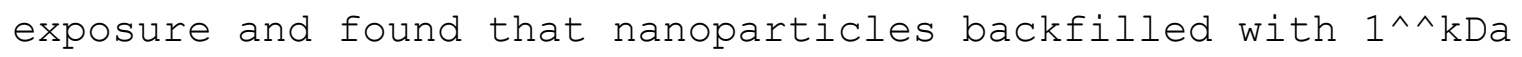
PEG displayed similarly high binding specificity for SKBR3 cells when compared to $2^{\wedge \wedge} \mathrm{kDa}$ backfill, while larger PEG (5 and $10^{\wedge} \mathrm{kDa}$ ) produced a drastic loss in binding specificity

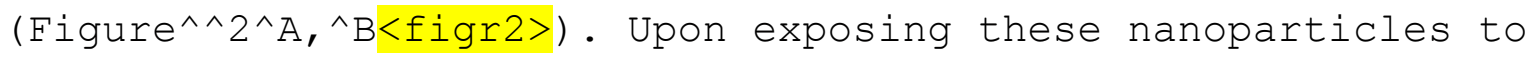
human serum, all of the PEG groups (1, 2, 5, and $\left.10^{\wedge \wedge} \mathrm{kDa}\right)$ used to backfill the nanoparticle surface showed a similar capacity to reduce serum-protein adsorption (Figure^^S5B). Qualitative (Figure^^S5C) and quantitative (Table^^S1) determination of ligand density on these nanoparticles showed similar amounts of conjugated Herceptin per particle, thus suggesting that the ligands are stable on the gold nanoparticle surface and that differences in the binding specificity are attributed to PEG length. Furthermore, nanoparticles backfilled with low molecular-weight<?><?>0k?<?><? $>$ PEG were able to retain high specificity for ErbB2<m+> cells, while this specificity is lost when larger PEG groups are used. Since a 5^^kDa linker was used for the conjugated Herceptin, anything equivalent or higher can potentially sterically hinder the Herceptin from binding to the target. $<$ ? $><$ ? $>$ Sentence ok? $<$ ? $><$ ? $>$ This suggests that although the binding specificity can be improved through PEG backfilling, it is also important to choose the backfill length based on the ligand linker length. $<$ ? $><$ ? $>$ Sentence ok? $<?><?>$

While it is know that PEG is an effective antifouling polymer for nanoparticles, a better understanding of the PEGylation process is key to ensuring that the surface chemistry is appropriate for biomedical use. Our results demonstrated that the although PEG can facilitate the reduction of nonspecific binding to cancer cells, this process is not as simple as putting PEG on the surface, since the chain length of the backfilled PEG has to be less than that of the ligand linker in order to not interfere with the binding of the biorecognition molecule to its target receptor (Figure^^ $2^{\wedge} C<x f i g r 2>$ ). PEG backfilling reduces the number of 
proteins that bind to the nanoparticle surface and prevents the serum proteins from sterically blocking the binding of the biorecognition molecule to the receptor target. This strategy improves the specificity of the nanoparticle conjugates and allows them to be used for selectively recognizing diseased cells in a serum rich environment.

Received: October 30, 2013

Revised: December 9, 2013

Published online: $<$ ? $><$ ? $>$

$<$ lit $1><$ lit_a $><j n l>M . \wedge \wedge E$. Davis, Z.^^G. Chen, D.^^M. Shin, Nat. Rev. Drug Discovery 2008, 7,771--782</jnl>; $<$ lit_b $><j n l>D \cdot{ }^{\wedge} \mathrm{L} \cdot{ }^{\wedge} \mathrm{J}$. Thorek, A.^^ $\mathrm{K}$. Chen, J. Czupryna, A. Tsourkas, Ann. Biomed. Eng. 2006, 34, 23--38</jnl>; $<$ lit_c $><j n l>D . \wedge \wedge$ G. Georganopoulou, L. Chang, J. Nam, C.^^s. Thaxton, E.^^J. Mufson, W.^^L. Klein, C.^^A. Mirkin, Proc. Natl. Acad. Sci. USA 2004, 101, 2273--2276</jnl>;

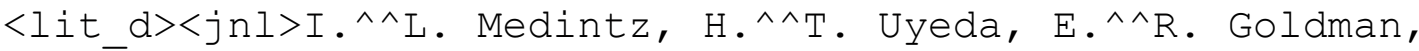
H. Mattoussi, Nat. Mater. 2005, 4, 435--446</jnl>.

$<$ lit $2><$ lit_a $<<j n l>D \cdot{ }^{\wedge}$ B. Kirpotin, D.^^ C. Drummond, Y. Shao, M.^^R. Shalaby, K. Hong, U.^^B. Nielsen, J.^^D. Marks, C.^^C. Benz, J.^^W. Park, Cancer Res. 2006, 66, 6732-$6740</ j n l>$; <lit_b $><j n l>G$. Wu, W. Yang, R.^^F. Barth, S. Kawabata, M. Swindall, A.^^K. Bandyopadhyaya, W. Tjarks, B. Khorsandi, T.^^E. Blue, A.^^K. Ferketich, et^^al., Clin. Cancer Res.2007, 13, 1260--1268</jnl>.

<lit3 $><$ lit_a $><j n l>J . \wedge \wedge D$. Hood, M. Bednarski, R. Frausto, S. Guccione, R.^^A. Reisfeld, R. Xiang, D.^^A. Cheresh, Science 2002, 296,2404--2407</jnl>; <lit_b $><j n l>D$. Simberg, T. Duza, J.^^H. Park, M. Essler, J. Pilch, L. Zhang, A.^^M. Derfus, M. Yang, R.^^M. Hoffman, S. Bhatia, et^^al., Proc. Nat1. Acad. SCi. USA 2007, 104, 932-$936</$ jnl $>$. 
$<$ lit $4><$ lit_a $><j n l>0 . \wedge \wedge C$. Farokhzad, S. Jon, A. Khademhosseini, T.^^N.^^T. Tran, D.^^A. Lavan, R. Langer, Cancer Res. 2004, 64, 7668--7672</jnl>; <lit_b><jnl>V. Bagalkot, L. Zhang, E. Levy-Nissenbaum, S. Jon, P.^^W. Kantoff, R. Langer, ${ }^{\wedge}{ }^{\wedge}{ }^{\wedge}$. Farokhzad, Nano Lett. 2007, 7, 3065--3070</jnl>.

<lit5 $><$ lit_a $><j n l>A . \wedge \wedge S$. Pitek, D. O'Connell, E. Mahon, M.^^P. Monopoli, F. Baldelli^^Bombelli, K.^^A. Dawson, PloS one 2012, 7, e40685</jnl>; <lit_b><jnl>C.^^D. Walkey, J.^^B.

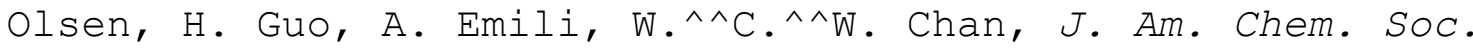
2012, 134, 2139--2147</jnl>.

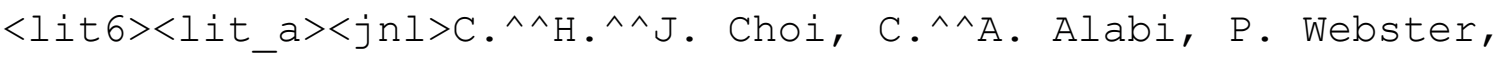
M.^^E. Davis, Proc. Natl. Acad. SCi. USA 2010, 107, 1235-$1240</ j n l>$; <lit_b><jnl>R.^^k. Jain, T. Stylianopoulos, Nat. Rev. Clin. Oncol. 2010, 2, 653--664</jnl>;

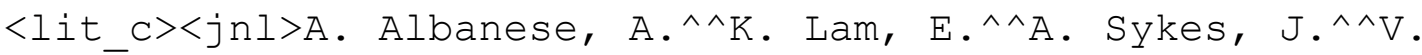

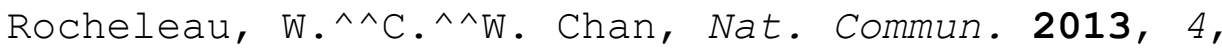
$2718</$ jnl $>$.

$<$ lit $7><$ lit_a $><j n l>W$. Jiang, B.^^${ }^{\wedge} \mathrm{Y} \cdot{ }^{\wedge} \mathrm{S} . \mathrm{Kim}, \mathrm{J} \cdot{ }^{\wedge}{ }^{\wedge} \mathrm{T}$. Rutka, W.^^C.^^W. Chan, Nat. Nanotechnol. 2008, 3, 145--150</jnl>; $<$ lit_b $><j n l>$ P. Decuzzi, M. Ferrari, Biomaterials 2007, 28, $2915--2922</ j n l>$.

$<$ lit $8><$ lit_a $><j n l>I$. Lynch, K.^^A. Dawson, $<$ ? $><$ ? > Nano today $<$ ? $><$ ? $>$ 2008, 3, 40--47</jnl>; <lit_b $><j n l>M$. Lundqvist, J. Stigler, G. Elia, I. Lynch, T. Cedervall, K.^^A. Dawson, Proc. Natl. Acad. SCi. USA 2008, 105, 14265$-14270</$ jnl $>$.

<lit9><jnl>S. Tenzer, D. Docter, J. Kuharev, A. Musyanovych, V. Fetz, R. Hecht, F. Schlenk, D. Fischer, K. Kiouptsi, C. Reinhardt, et^^al., Nat. Nanotechnol. 2013, 8, 772-$781</ j n 1>$.

<it10 $><$ lit_a $><j n l>A$. Salvati, A.^^S. Pitek, M.^^P. Monopoli, K. Prapainop, F.^^B. Bombelli, D.^^R. Hristov, P.^^M. Kelly, C. Åberg, E. Mahon, K.^^A. Dawson, Nat. Nanotechnol. 2013, 8, 137--143</jnl>; <lit_b><jnl>V. Mirshafiee, M. 
Mahmoudi, K. Lou, J. Cheng, M.^^L. Kraft, Chem. Commun. 2013, 49, 2557--2559</jnl>.

<lit1l><lit_a $><j n l>R$. Gref, M. Lück, P. Quellec, M. Marchand, E. Dellacherie, S. Harnisch, T. Blunk, R. Müller, Colloids Surf. B 2000, 18, 301--313</jnl>; <lit_b><jnl>K. Knop, R. Hoogenboom, D. Fischer, U.^^s. Schubert, Angew. Chem. 2010, 122, 6430--6452; Angew. Chem. Int. Ed. 2010, 49, 6288-6308</jnl>; <lit_C><jnl>A. Vonarbourg, C. Passirani, P. Saulnier, J.-P. Benoit, Biomaterials 2006, 27, 4356-$4373</ j n l>$.

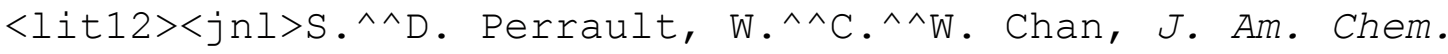
Soc. 2009, 131, 17042--17043</jnl>.

<lit13><jnl>M.^^^C. Daniel, D. Astruc, Chem. Rev. 2004, 104, $293--346</ j n l>$.

<lit14><jnl>G.^^F. Paciotti, L. Myer, D. Weinreich, D. Goia, N. Pavel, R.^^Е. McLaughlin, L. Tamarkin, Drug Delivery 2004, 11, 169--183</jnl>.

<lit15><jnl>C.^^A. Hudis, N. Engl. J. Med. 2007, 357, 39-$51</ j n l>$.

<lit16><jnl>L. ${ }^{\wedge}{ }^{\wedge} \cdot{ }^{\wedge} \mathrm{T} \cdot$ Chou, $\mathrm{W} \cdot{ }^{\wedge}{ }^{\wedge} \mathrm{C} \cdot{ }^{\wedge}{ }^{\wedge} \mathrm{W}$. Chan, Adv. Healthcare Mater. 2012, 1, 714--721</jnl>.

<lit17><jnl>N. Chattopadhyay, Z. Cai, J. Pignol, B. Keller, E. Lechtman, R. Bendayan, R.^^M. Reilly, Mol. Pharm. 2010, 7, $2194--2206</ j n l>$.

<lit18><jnl>R.^^A. Sperling, W.^^J. Parak, Philos. Trans. R. Soc. London Ser. A 2010, 368, 1333--1383</jnl>.

<lit19><other>ThermoScientific method for calculating dye protein (F/P) molar ratios can be found under <url>http://www.piercenet.com/files/TR0031-Calc-EPratios.pdf</url>, n.d<?><?>not determined? not dated $<$ ? $><$ ? $></$ other $>$.

$<$ lit20 $><j n l>G$. Frens, Nature 1973, 241, 20--22</jnl . 


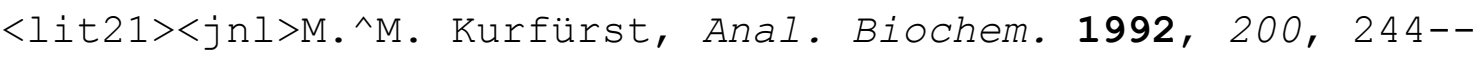
$248</$ jnl $>$. $<$ ?><? $>$ Please refer to Refs. [19--20] in the text. $<?><?>$

Figure^^1 Binding specificity is reduced by serum-protein adsorption but this effect is alleviated by PEG backfilling. A)^^Herceptin (1) was initially conjugated to Alexa Fluor 647 (AF647; 2) through amine-reactive succinimidyl ester chemistry, followed by a second conjugation step with orthopyridyldisulfide polyethyleneglycol N-hydroxysuccinimide ester (OPSS-PEG-NHS ester, 3) to generate OPSS-PEG-HerceptinAF647 (4). The surfaces of the 50^^nm gold nanoparticles were functionalized with (4) as well as methoxypolyethyleneglycol (mPEG, 6) through thiol coordination. Alternatively, the nanoparticles were treated with human serum. The molecular weight $<$ ? $><$ ? > ok? $<$ ? $><$ ? $>$ of the PEG groups are denoted by $n$ and $m$, where $n^{1 \wedge} 5^{\wedge} \mathrm{kDa}$, and $m=1,2,5$, or 10^^kDa. B)^^Herceptin-conjugated nanoparticles were incubated with SKBR3 or MCF7 cells in addition to a 50-fold molar excess of unlabeled Herceptin (competition) to assess the specificity of ErbB2 receptor binding. C)^^The ErbB2-receptor binding specificities of these nanoparticles were quantified through flow cytometry. Red and blue lines represents cells incubated in the absence and presence of competitive Herceptin molecules, respectively. Herceptin-conjugated nanoparticles without (D) and with (E) PEG were exposed to serum and subsequently incubated with SKBR3 and MCF7 cells.<?><?>Sentence ok?<?><?> Error bars represent the standard error, where $n=3$. The $x$-axis indicates the amount of targeted nanoparticles binding on cells, and the y-axis represents the normalized number (all events included) of cells counted by flow cytometer. $<$ ?><?>Could you clarify the flow cytometry plots, it looks like there is fluorescence intensity on both axes?I apologize for the unclear statement. The $\mathrm{x}$-axis represents the fluorescence intensity in the FL-4 channel (661/16nm emission), indicating the amount of targeted 
nanoparticles binding to the cells. The y-axis is the normalized number (all events included) of cells that was counted by the flow cytometer. $<$ ? $><$ ? $>$

Figure^^2 The molecular weight<?><?>0k? $<$ ? $><$ ? $>$ of the PEG used for backfilling affects the binding specificity.<?><?>Sentence ok?<?><?> Herceptin-conjugated gold nanoparticles with 1, 5, and $10^{\wedge} \mathrm{kDa}$ PEG backfill were incubated with SKBR3 and MCF7 cells prior to (A) and post (B) exposure to human serum. Red and blue lines represents cells incubated in the absence and presence of competitive Herceptin molecules, respectively. Error bars represent the standard error, where $n=3$. The binding specificity is dependent on serum-protein adsorption and the molecular weight of the PEG used for backfilling.<?><?>Sentence ok?<?><?> When the nanoparticles are exposed to a serum-rich environment, the surface coverage of the PEG backfilling and serum-protein adsorption determines the targeting specificity of the nanoparticle. The size of the arrows indicates the likelihood of interaction of the nanoparticles with the cells, with red indicating an alternative (non-ErbB2) pathway and green indicating the ErbB2-mediated pathway. The $x$-axis indicates the amount of targeted nanoparticles binding on cells, and the y-axis represents the normalized number (all events included) of cells counted by flow cytometer. $<$ ? $><$ ? $>$ Please clarify the flow cytometry plots (see Figure^^1). $\langle$ ? $><$ ? $>$ 